\title{
La integración regional como proceso contrahegemónico: aportes teóricos acerca de la Unasur como integrador ${ }^{\star}$
}

Regional integration as a counter-hegemonic process: Theoretical contributions about USAN as inclusive

\begin{abstract}
A integração regional como um processo contra hegemônico: Contribuições teóricas sobre a Unasul como integrador
\end{abstract}

Recibido el 29 de agosto de 2016. Aceptado el 21 de marzo de 2017

\author{
Angélica Nieto Roa** \\ Colombia \\ María Camila Malagón*** \\ Colombia
}

Para citar este artículo:

Nieto Roa, Angélica; Malagón,

María Camila; Yepes Lugo,

Cristian y Castro Castell, Ofelia

(junio, 2017). La integración

regional como proceso

contrahegemónico: aportes

teóricos acerca de la Unasur

como integrador. Ánfora, 24(42),

95 - 115. Universidad Autónoma

de Manizales. ISSN 0121-6538.

\section{Resumen}

Objetivos: determinar la dinámica del proceso contrahegemónico que surgió con la integración regional, a partir del caso de estudio de la Unión Suramericana

\footnotetext{
*Este artículo se deriva de la investigación en curso "Integración regional como proceso contra hegemónico: Caso de estudio Unasur". La investigación es liderada por el Semillero Se-koiné de la Facultad de Ciencias Económicas y Sociales de la Universidad de La Salle. La investigación se inició en febrero de 2016

** Estudiante de Negocios y Relaciones Internacionales, Universidad de la Salle. Pasante de investigación Semillero Se-koiné facultad de ciencias económicas y sociales, Universidad de la Salle. Correo electrónico: anieto02@unisalle.edu.co

*** Estudiante Negocios y Relaciones Internacionales, Universidad de la Salle. Pasante de investigación Semillero Se-koiné facultad de ciencias económicas y sociales, Universidad de la Salle. Correo electrónico: mmalagon22@unisalle.edu.co

**** Magíster en Relaciones y Negocios internacionales. Doctorando en industria y organizaciones. Profesor e investigador de la Universidad de la Salle. Correo electrónico: cyepes@unisalle.edu.co

Magíster en gestión de organizaciones. Magíster en comercio internacional y logística integral. Profesora e investigadora de la Universidad de la Salle. Correo electrónico: ofcastro@unisalle.edu.co
} 
de Naciones, (Unasur). Metodología: se utilizó un enfoque cualitativo que permitió discutir categorías de análisis, tales como Integración Regional, Contrahegemonía y Unasur. Esto, a partir de la revisión teórica que tiene como base el análisis del posicionamiento del regionalismo, desde el enfoque de los gobiernos de izquierda en América del Sur, cuyos objetivos han buscado contrarrestar el dominio hegemónico en la región. Resultados: se concluye que Unasur surgió como una expresión del regionalismo posliberal, liderada por los entonces presidentes Chávez, Lula Da Silva, y Kirchner. Aunque actualmente se encuentra en un estado de incertidumbre, Unasur se identifica como un instrumento político, que garantiza la autonomía de sus Estados miembros; ello, a través de iniciativas de cohesión regional, la protección política de los gobiernos afines y la expansión de la idea del cambio social. Es decir, se ha perfilado como la antítesis de la influencia de los Estados Unidos en la región. Conclusiones: la nueva estructura de política exterior planteada por América del Sur en el siglo XXI se caracteriza por una fuerte influencia del regionalismo postliberal; un ejemplo, es la aparición de integraciones como la de Unasur, que propende por la autonomía regional con un enfoque contrahegemónico.

Palabras Clave: Contrahegemonía; Integración; Política; Suramérica.

\begin{abstract}
Objective: to determine the dynamics of the counterhegemonic process that emerged with the regional integration based on the case study of the Union of South American Nations (USAN). Methodology: a qualitative approach was used which allowed discussing analysis categories, suchas Regional Integration, Counter-hegemony and USAN. What was said before, based on the theoretical review, is that it is related to the analysis of the regionalism position, from the approach of leftist governments in South America, whose objectives have been finding a way to counter the hegemonic domination in the region. Results: It is concluded that USAN emerged as a way of expression from the post-liberal regionalism, led by the presidents, back then, Chavez, Lula Da Silva, and Kirchner. Although, USAN is currently in a state of uncertainty, it is identified as a political instrument, guaranteeing the autonomy of its states through the regional cohesion efforts, the political protection of allied governments and the expansion of the social change idea. That is to say, it has been defined as the antithesis of the influence from the United States in the region. Conclusions: the new structure of the foreign policy, raised by South America in the 21st century, is characterized by a strong influence of the post-liberal regionalism. For example, the emergence of integrations such as USAN, which works for the regional autonomy with a counterhegemonic approach.
\end{abstract}


Key words: Counter-hegemony; Integration; Politics; South America.

\section{Resumo}

Objetivo: determinar a dinâmica do processo contra-hegemônico que apareceu com a integração regional a partir do estudo do caso da União Sul-Americana de Nações (Unasul). Metodologia: usou-se um enfoque qualitativo o qual permitiu discutir categorias de análise, como a integração regional, contra-hegemonia e Unasul. Isto, a partir da revisão teórica que se baseia na análise do posicionamento do regionalismo, a partir da perspectiva dos governos de esquerda na América do Sul, cujos objetivos têm procurado contrariar o domínio hegemônico na região. Resultados: concluiu-se que a Unasul surgiu como uma expressão do pós regionalismo liberal, liderado pelos presidentes da época Chávez, Lula da Silva e Kirchner. Embora atualmente se encontra em um estado de incerteza, a Unasul é identificada como um instrumento político, que garante a autonomia dos seus Estados-Membros; Assim, através de iniciativas de coesão regional, proteção política dos governos relacionados e a expansão da ideia de mudança social. Ou seja, tem sido perfilado como a antítese da influência dos EUA na região. Conclusões: a nova estrutura de política externa exposta pela América do Sul no século XXI é caracterizada por uma forte influência do pós - regionalismo liberal; Um exemplo é o surgimento de integrações como a Unasul, que visa à autonomia regional com um enfoque hegemônico.

Palavras-chave: Contra-hegemonia; Integração; Política; América do Sul. 


\section{Introducción}

A partir del siglo XIX, algunos países de América Latina iniciaron procesos de integración regional. Hechos históricos como el Manifiesto de Cartagena de Simón Bolívar en 1812, el Congreso de Panamá en 1826 y el establecimiento de la Asociación Latinoamericana de Libre Comercio (Alalc) en 1960, dieron lugar a la fundación de la Unión de Naciones Suramericanas (Unasur) en 2008. El constante intento por consolidar el bloque regional ha estado asociado a una variedad de factores, entre ellos el rol de los Estados Unidos en la región y su oposición a la configuración de un bloque regional que haga contrapeso a sus intereses en el llamado "patio trasero" (Martínez, 2006).

Una de las épocas más relevantes para el regionalismo latinoamericano fue la de los años 90, en la que se promovió la concertación en términos de política exterior, cooperación ambiental, cuestiones de seguridad regional y los movimientos y sindicatos. En efecto, la integración económica simple se transforma en una integración de consulta política dinámica, a través de instituciones de cooperación y construcción de políticas regionales en diversos campos. Así, dicha integración se configura con el fin de promover la competitividad internacional de los países miembros y aumentar su peso en materia de negociación en comparación con los países industrializados (Sanahuja, 2007, p. 77).

En este sentido, Estados Unidos diseñó un plan para la integración política y económica regional y la liberalización de las economías dependientes, aunque esto no es considerado como un objetivo exitoso de la integración. La llamada Área de Libre Comercio de las Américas (Alca) se inició en la Cumbre de las Américas, a través de la cual los jefes de Estado, de los 34 países de la región, acordaron la creación de un área de libre comercio en la que las barreras comerciales y la inversión podrían ser eliminadas progresivamente (Vivas-Eugui, 2003).

Sin embargo, las nuevas interacciones y dinámicas de los países de la región alejan gradualmente a América Latina del status quo en las últimas décadas. Dicho proceso genera el retorno de la política como un elemento necesario en términos de las relaciones internacionales y la disminución de la intervención de los Estados Unidos y las instituciones interamericanas en los procesos que involucren a los Estados sudamericanos (Bonilla, 2010).

El escenario de los procesos de integración en América del Sur ha presentado importantes cambios en el transcurso del tiempo. A partir del año 2000, las iniciativas con mayor trayectoria se enfocaron en la liberalización del comercio; 
el Mercado Común del Sur (Mercosur) y la Comunidad Andina (CAN) perdieron centralidad en relación con nuevos eventos. Dichas iniciativas no lograron consolidar una regionalización que incrementara la interdependencia en los países; por consiguiente, fue imposible reformular el comercio intrarregional. Estos procesos, se basaron en el modelo de economía abierta; sin embargo, con el posicionamiento de gobiernos progresistas el modelo fue sustituido por políticas más cercanas al desarrollismo (Gomes, 2012).

De forma paralela, Brasil promovió la integración de América Latina, en la búsqueda de la creación de un espacio de desarrollo integrado para los países miembros; por eso, se conformó la Unión de Naciones Suramericanas (Unasur) en 2004, la cual fue constituida oficialmente en el 2008.

El presente artículo explica la forma en que la integración regional, en el caso de Unasur, se puede caracterizar como un proceso contrahegemónico. Para ello, se ha identificado una amplia bibliografía en temas relacionados con la historia y evolución de la integración regional en América Latina, el rol de Brasil en términos de liderazgo regional y la conformación de bloques políticos y económicos.

Se han llevado a cabo estudios concernientes a la creación y desarrollo de la Unión de Naciones Suramericanas, a través de estudios comparados con distintos procesos regionales como Mercosur y Alba (Alcoceba, 2010, Behar, 2001, Bonilla, 2010, Briceño, 2013, Diamint, 2013, Morra, 2007, Navarro, 2013). Sin embargo, se observa la falta de estudios y análisis en relación con los fenómenos contrahegemónicos, su aplicabilidad en las coyunturas y desafíos actuales y su proceso y evolución en América del Sur.

El objetivo de este artículo, entonces, es explicar la manera cómo la integración regional ha emergido como un proceso contra-hegemónico, a partir del caso de estudio de la Unión Suramericana de Naciones. Esta investigación constituye una base útil para el estudio de este fenómeno, ya que permite el análisis de los hechos y las decisiones históricas de los países latinoamericanos que pudieron ser el desencadenante de sucesos determinantes para la posición de los gobiernos de la región con respecto a la hegemonía norteamericana, incluyendo aspectos como las relaciones bilaterales y multilaterales en las áreas de la diplomática, comercial y de cooperación.

A partir del análisis de los aportes teóricos de la Unasur, como proceso integrador, y de la importancia que dichos aportes tienen para la comprensión 
de las dinámicas regionales, resulta interesante indagar por el auge de la Unión de Naciones Suramericanas como un proceso contrahegemónico. Lo anterior, mediante el estudio de los acontecimientos más relevantes desde su creación y el análisis del discurso de los líderes regionales, lo cual resulta fundamental para lograr un entendimiento completo del proceso contra hegemónico anteriormente reseñado.

\section{Metodología}

Es una investigación de tipo cualitativo cuyo método consistió en la revisión documental sobre las causas coyunturales del nacimiento de la integración regional y el proceso de los eventos que la caracterizan como un ente antagónico a las políticas e ideologías hegemónicas instauradas en la región.

La metodología se aplicó en dos fases: primero, se realizó una revisión de literatura a través del rastreo bibliográfico en libros, documentos oficiales y bases de datos especializadas, sobre los procesos de integración regional en América Latina y Movimientos Sociales. A partir de la clasificación de la información de treinta y cinco documentos provenientes de diferentes fuentes en fichas bibliográficas, se procedió al análisis de las categorías de investigación: integración regional, Unión de Naciones Suramericanas (UNASUR) y contra hegemonía.

Teniendo en cuenta la información sintetizada en fichas de trabajo, durante la segunda fase, se ponen en discusión los referentes teóricos de cada una de las categorías anteriormente mencionadas. A través del método hermenéutico se realizó una lectura diacrónica, la cual permitió establecer las conclusiones con respecto a los resultados. La anterior clasificación, se evidencia en la siguiente tabla, que presenta la cantidad de documentos revisados por fuente: 
Tabla 1. Clasificación de las fuentes de búsqueda, según las categorías

\begin{tabular}{|c|c|c|c|c|c|}
\hline & Libros & Revistas & Tesis & $\begin{array}{c}\text { Bases de } \\
\text { Datos }\end{array}$ & Sitios Web \\
\hline Integración Regional & 5 & 7 & 0 & 1 & 0 \\
\hline Unasur & 1 & 6 & 3 & 1 & 2 \\
\hline Contrahegemonía & 6 & 3 & 0 & 0 & 0 \\
\hline
\end{tabular}

Fuente: elaboración propia

\section{Resultados}

Se evidenció que las nuevas dinámicas de integración regional en América del Sur y la ola de gobiernos de izquierda en la mayoría de los Estados miembros, han buscado contrarrestar el dominio hegemónico en la región. El desarrollo de la Unión Suramericana de Naciones como un bloque político y económico, se ha perfilado como la antítesis de la influencia de los Estados Unidos. El constante intento por solidificar el bloque regional se asocia a una mirada de factores externos e internos.

Sin embargo, dicha "izquierda latinoamericana" eventualmente enfrentó la pérdida de influencia en la región, lo que conllevó a algunos gobiernos a un acercamiento a la derecha, para crear un nuevo balance de poder que deterioraría la legitimidad -en este caso- de Unasur. Lo anterior, se atribuye a la llamada "ineficacia de la izquierda", que se ha visto envuelta en una serie de escenarios de inestabilidad política.

Los resultados se presentan así: como primer elemento se expondrán los elementos más relevantes de la integración regional en América del Sur, a partir de los enfoques obtenidos de distintos autores se pondrá en discusión el análisis de los mismos. Posteriormente, se presentará el marco de creación y desarrollo de la Unión de Naciones Suramericanas, su actuar político y su comprensión académica. Por último, se desarrolla el termino de contra hegemonía en la región suramericana, teniendo como base la visión gramsciana, la cual permite el análisis del auge de Unasur como un proceso contra hegemónico. 


\section{La integración regional en américa del sur}

Según Behar (2001) las características e implicaciones de la integración regional han sido abordadas en la literatura especializada desde distintas perspectivas. Enfoques que permiten abarcar aspectos macroeconómicos y participación de agentes sociales en los procesos de integración. En su orden, como lo afirma Viner (1950; citado por Behar, 2001) la integración macroeconómica se basa en las uniones aduaneras y los efectos que éstas generan en la creación o desviación de comercio; parte además de una hipótesis basada en el comercio internacional, que deja en un segundo plano fenómenos como economías de escala y diseños comerciales discrepantes enmarcados en la teoría clásica de las ventajas competitivas (Behar, 2001, p. 6).

El análisis planteado por Viner(1950; citado por Behar, 2001) y la incorporación de las formas de competencia imperfecta en el estudio integracionista, permitió abordar el estudio acerca de bloques económicos regionales desde nuevas perspectivas. Sin embargo, se excluyen las implicaciones del desarrollo en áreas monetarias y financieras, especialmente en el caso Latinoamericano donde han cobrado importancia en el debate actual de integración. El primer enfoque se basa en la liberalización de la cuenta de capitales de la balanza de pagos, la desregulación de la inversión extranjera directa y las negociaciones propuestas por Estados Unidos con el fin de conformar una zona de libre comercio hemisférica.

El segundo enfoque aborda la participación de actores de la integración; como afirma Haas (1958), el debate se desarrolla bajo tres ejes teóricos: el carácter de la participación, los efectos de la integración y los roles que desempeñan en el proceso integracionista.

En este sentido, tanto los actores como el propio proceso de integración no pueden llevar a cabo sus procesos per se; es necesario el establecimiento de una relación mutua. Posterior a dicho análisis, y como consecuencia, el enfoque que se otorgó a los efectos de la integración sobre los actores, dejaron de ser conceptualizados como factores que afectan directamente los procesos de integración.

Bajo la noción de la Teoría de la Dependencia, se le dio a la integración un enfoque netamente económico; según Behar (2001) "los actores "malos" (p.1) por definición lograrán imponer sus intereses en desmedro de otros actores "buenos". A partir de ello, surge una perspectiva en la cual el eje de participación cobra 
mayor relevancia; en efecto, emergió un cuestionamiento central para el debate: ¿Quiénes son los actores centrales de la integración? El análisis planteado realiza un mayor énfasis en los actores empresariales (empresas transnacionales, grupos económicos y /o asociaciones industriales) sin dejar de lado la importancia de gobiernos y de las tecnoburocracias regionales. Cabe resaltar también los efectos de la integración en términos de modificar el contenido del proceso, los intereses del mismo y la percepción mutua de los diversos agentes.

Con base en el desarrollo de los enfoques de la integración regional, resulta preponderante el análisis de dicho proceso en la región Suramericana. La integración latinoamericana ha presentado una nueva fase de su desarrollo, en la que se observan nuevas realidades. Tomando como referencia la coyuntura de la última década, Sanahuja (2010), Motta Veiga y Ríos (2007) introducen el concepto de "regionalismo posliberal" en América del Sur. Según estos autores, el trance se ha dado desde el "regionalismo abierto" latente en los años 90 a través de la creación del Mercosur y la Comunidad Andina, hasta la emergencia del "regionalismo posliberal" cuyas expresiones se enmarcan con la Unasur y el ALBA- TCP.

Por otra parte, Rigirozzi (2010) hace uso del término "regionalismo poshegemónico" que representa una ruptura del discurso hegemónico durante la década de 1990, el cual asociaba el regionalismo a los procesos de globalización; así, el regionalismo se percibía como una defensa a los procesos globales. A su vez, Briceño (2013) coincide en que el actual y complejo escenario de integración regional en América Latina, tiene una relación entre los ejes de integración realmente existentes: el eje de regionalismo abierto, el eje revisionista y el eje antisistémico, el cual propone políticas propias del modelo de regionalismo social y del regionalismo productivo, que no se concibe como un modelo no capitalista.

Retomando el contexto histórico, el eclipse del regionalismo abierto, basado en una agenda de liberalización comercial y una óptica economicista, se debe al posicionamiento de la izquierda política en muchos gobiernos de la región, los cuales rechazan los esquemas económicos tradicionales, la debilidad institucional y el estancamiento del comercio. Ejemplo de ello, ha sido el tardío proceso de la CAN y Mercosur para crear proyectos de cooperación política y judicial en pro de hacerle frente a la delincuencia organizada (Alcoceba, 2010).

Uno de los factores decisivos para la aparición de los proyectos políticos del ALBA-TCP y Unasur, según Sanahuja (2010), ha sido la creación de la nueva agenda de seguridad de Estados Unidos, derivada de los acontecimientos del 11 
de septiembre del 2001, en la que América Latina queda en un segundo plano para el país potencia. Dicha "desatención” genera una evolución en la autonomía de los gobiernos, la cual incluye temas económicos, de seguridad, defensa política exterior y de desarrollo en cada nación. "Este nuevo regionalismo latinoamericano es el que más ha avanzado, buscando desafiar al sistema interamericano (OEA, TIAR) con el objetivo de sustituirlos" (Gratius, 2011). De acuerdo con lo planteado por Saraiva (2012), ni el Gobierno Bush ni la administración Obama, lograron concretar una política orientada para América del Sur. Además, los gobiernos de carácter antiliberal electos en la región han contribuido a profundizar la distancia y debilitar los lazos históricos que la unían con el norte.

Ahora, la etapa post-liberal y post hegemónica del regionalismo en América del Sur se encuentra bajo el marco de distintos ejes de integración regional y modelos económicos. Según Pereira (2010), la integración de América del Sur se basa en tres pilares fundamentales:

- Cooperación política, social y cultural

- Integración Comercial, Financiera y económica

- Integración física, energética y de comunicaciones

Es así como Unasur nace como un proyecto regional, basado en la consolidación de una región articulada políticamente a través de prácticas de participación y una nueva ciudadanía. Por eso, es preponderante el estudio de la creación y desarrollo de dicho proyecto regional, el cual, como lo afirma Braudel (1979 citado por Narvaja, 2012), se entiende como "La construcción, entonces, de un imaginario colectivo que movilice las pasiones políticas fraternas para enfrentar externa e internamente las asimetrías, diferencias y exclusiones generadas por la geografía discriminatoria propia del capitalismo” (p. 15).

\section{Unión de Naciones Suramericanas (Unasur)}

Los inicios de la Unasur se remontan al año 2000, a partir de las reuniones de presidentes de América del Sur, en las que, de acuerdo con Insignares (2013), se vio la necesidad de establecer una continuidad geográfica y una comunidad de valores que concurrieran en una agenda común de desafíos y oportunidades, lo cual se materializó con la Declaración de Cusco de 2004, en el marco de la III Reunión de Presidentes de América del Sur. 
A partir de su creación, la Unasur ha representado un papel fundamental en los eventos de la región; ha sido reconocida como un canal de diálogo entre las naciones, actuando de mediadora en casos como la política doméstica en Bolivia y relaciones fronterizas entre Venezuela y Colombia. Existen dos diálogos interregionales estructurados en su marco América del Sur-África y América del Sur-países árabes y, en el caso de la Unión Europea, ya hay un reconocimiento de los formuladores europeos sobre la importancia de establecer un diálogo UEUnasur.

Se puede considerar, entonces, una novedad con un perfil diferente de otras iniciativas, que acomoda diferencias y que puede hacer una importante contribución a la consolidación de una gobernanza regional (Gomes, 2012).

Con el surgimiento de un nuevo escenario en la región, en el que se priorizan los campos de integración y cooperación; el objetivo de la creación de la Unión de Naciones Suramericanas (Unasur) sería el de garantizar la autonomía de los Estados miembros en iniciativas intrarregionales. Como lo afirma Gomes (2012) "En términos económicos, por no tener los compromisos inherentes a una experiencia de integración económica, puede acomodar diferentes iniciativas subregionales como el Mercosur y la CAN” (p. 95).

La Unión de Naciones Suramericana, inició en el año 2004 como la Comunidad Sudamericana de Naciones (CSN) para la que los países miembros ${ }^{1}$ conformaron una agenda común en los años 2005 y 2006; incluyeron materias como el diálogo político, integración física, medio ambiente, integración energética, mecanismos financieros suramericanos y la promoción de la cohesión social, para posteriormente otorgarle el nombre de Unión de Naciones Suramericanas (Unasur) en el 2007 durante la cumbre energética suramericana.

Kacowicz, (2008; citado por Martínez Garnelo, 2013) afirma que la Unasur se basa en iniciativas económicas y políticas, que incluyen la integración geopolítica por medio del fortalecimiento regional de las comunicaciones, rutas y energía, acuerdos de libre comercio integrando a Mercosur con la CAN y con un deber generalizado en asuntos de cooperación para superar la pobreza e inseguridad. Lo anterior, con el objetivo de mejorar la competitividad de Sur América y su capacidad de integración en la economía mundial, además de promover el desarrollo sostenible de los países de la región.

1 Brasil, Argentina, Bolivia, Perú, Chile, Ecuador, Venezuela, Colombia, Guyana, Paraguay, Surinam, Uruguay. 
Unasur tiene un perfil diferente de las demás iniciativas y se acerca más a un instrumento de gobernanza regional que a los patrones clásicos de integración; existía el imaginario de una unificación en las conductas regionales -hasta la reciente crisis de la izquierda- y constituyó un papel importante en las crisis de la región como los casos de política doméstica de Bolivia y relaciones fronterizas entre Venezuela y Colombia (Saraiva, 2012). Además, se le reconoce como un medio para el diálogo por otros actores, de ahí que existan dos diálogos interregionales constituidos en el marco de las cumbres de América del SurÁfrica (Asacof) $)^{2}$, América del Sur y países árabes (ASPA) ${ }^{3}$, por lo que es posible establecer que la Unasur sostiene un perfil enfocado hacia la gobernanza, la seguridad y el liderazgo regional.

Con base en lo anterior, se establece que desde su creación, Unasur ha dado prioridad a los objetivos de sus políticas, convirtiéndose así en un cuerpo capaz de hacer frente a los conflictos en la región. Asimismo, es caracterizada por el regionalismo en América Latina como una crítica al modelo neoliberal. En efecto, expresa su afinidad con una visión post-liberal basada en las respuestas de la región a las nuevas identidades (Sanahuja, 2010). Sin embargo, antagónicamente, Diamint (2013) plantea que a pesar de que la Unasur se abre vías para la unión política estratégica, no existe una forma de integración económica; más bien, existe la noción de que América del Sur se encuentra en construcción y se caracteriza por tener una narrativa donde hay pocas acciones formalizada; es decir, que no se ha creado un nuevo tipo de regionalismo, sino que más bien representa un proyecto político unificado.

Como todas las dinámicas de integración, Unasur enfrenta retos para lograr la consolidación de su discurso que, según Bermúdez (2011), son: 1) Contar con la disposición de los gobiernos miembros para alcanzar decisiones que hagan frente a las necesidades y problemáticas de la región; 2) trabajar por los intereses comunes, venciendo los ideales de los intereses particulares de los gobiernos; 3) encontrar los métodos a ejecutar para evitar que los conflictos y las problemáticas anteriormente evidenciadas en la CAN, Mercosur y OEA afecten o influencien su institucionalidad; y 4) agudizar los esfuerzos para conseguir los objetivos planteados de acuerdo al propósito de progreso de la integración.

\footnotetext{
2 Está integrado por 55 países de África y 12 de América del Sur, en su mayoría miembros respectivamente de la Unión Africana (UA) y de la Unión de Naciones Suramericanas (UNASUR).

3 Los países miembros del ASPA, son los 22 países Árabes que integran la Liga de Estados Árabes: Argelia, Bahréin, Comoras, Egipto, Emiratos Árabes Unidos, Iraq, Jordania, Kuwait, Líbano, Libia, Mauritania, Marruecos, Omán, Palestina, Qatar, Arabia Saudí, Somalia, Sudán, Siria, Túnez, Yemen, Yibuti, así como los 12 países que conforman UNASUR.
} 
Las contraposiciones existentes en referencia a la Unasur surgen en debate que evalúa su eficacia. Por eso, como Miranda (2011) y Saraiva (2009; citado por Diamint, 2013) aseguran, este organismo es "la primera alternativa netamente latinoamericana que intenta ir más allá de lo económico y da un paso más allá hacia lo político y geoestratégico".

En efecto, el ámbito de seguridad se ha considerado como el punto de convergencia para lograr procesos eficaces de diálogo entre los líderes de la región; además, ha encajado en el multilateralismo con una estrategia basada en la autonomía y la búsqueda de poder -en el caso de Brasil- a la aceptación de una manera pragmática de la percepción de la democracia como valor universal, lo que lleva a instaurar un consenso diplomático en el marco de la integración regional y las perspectivas de desarrollo.

Mientras Diamint (2013), aunque reconoce los avances político-estratégicos, afirma que no existe una forma de integración económica y esto deja a América del Sur bajo el imaginario de ser una región en construcción y estar caracterizada por ejecutar pocas acciones formalizadas; este planteamiento lleva a la conclusión de que no es un nuevo tipo de regionalismo. Y Borda (2012), considera que la capacidad de la Unasur para tomar decisiones vinculantes que generen cambios radicales en el comportamiento de los Estados miembros es débil, debido a que el proceso de toma de decisiones está determinado por el principio de unanimidad", respeto a la soberanía, la no injerencia y la autodeterminación; esto hace que el consenso en las resoluciones sea un objetivo difícil de conseguir al interior de un organismo conformado por diferentes ideologías y políticas.

Chávez (2010) plantea un desafío concerniente a la concertación de las políticas de desarrollo; incidiendo en el diseño, la discusión y la ejecución de las políticas globales. Las cuales permitirán a la región la conformación de un bloque geopolítico de relevancia en el sistema internacional, con el objetivo de emerger como un multilateralismo efectivo que represente sus intereses; pues en Sudamérica no existe una clara convergencia de prioridades entre naciones lo que significa una amenaza para el pleno desarrollo de la identidad en la región, al subordinar los intereses regionales a los intereses geopolíticos nacionales demostrando el predominio de la ideología sobre la convergencia regional.

4 Tratado de 2008, Artículo 12. 
Bermúdez (2011), de su parte, plantea que los desafíos de esta integración conciernen a aspectos más amplios que la sola necesidad estratégica de la región; asegura que la convergencia de intereses mutuos, por encima de los intereses particulares, sumada al compromiso de los gobiernos y la ejecución de mecanismos que "blinden" a Unasur de los conflictos creados por otras integraciones, es realmente lo necesario para que la Unión Suramericana de Naciones no sea simplemente parte de la retórica integracionista sin acciones concretas.

De acuerdo con lo anterior, la Unión Suramericana de Naciones ha sido definida como la "integración de la integración"; su objetivo ha sido vincular procesos de integración existentes por medio de criterios de convergencia basados en proyecciones sociales y valores culturales. Es decir, Unasur es el resultado de la suma entre "el acuerdo comercial entre la Comunidad Andina y Mercosur, además de la integración física en la región suramericana y la proyección de la integración energética" (Cano, 2010).

La creación de esta organización se dio, pues, con el fin de producir un equilibrio en el sistema internacional; ello, como oposición a la hegemonía de los Estados Unidos. La visualización de un líder regional permitiría contrarrestar el poder del "hegemón" y formar alianzas para superar su dominio. De este modo, América del Sur muestra signos de cambio en la relación con Estados Unidos, ya que a partir de la década de los noventa se ha direccionado a un "conjunto de transformaciones que facilitaron la globalización de los circuitos económicos" (Navarro, 2013, p. 5).

\section{Contrahegemonía en la región suramericana}

Como lo afirma Campione (2005), el concepto de hegemonía en un sentido amplio y complejo, ahonda el camino para un proyecto que no se inclinará a descubrir una sola clave de la sociedad existente para impugnarla desde allí, sino a visualizar una crítica global, articulada sobre la problemática de la lucha de clases, eludiendo, a su vez, la tentación de subsumir en el plano de las relaciones de propiedad y el manejo del aparato coercitivo estatal.

En contraste, se aborda el concepto contrahegemonía que se refiere a la creación de un ente social que pueda enfrentar de manera exitosa al poder establecido; debe construir una expresión política, unitaria, y plantear una alternativa general, tanto en los temas inherentes a la agenda nacional como 
en el nivel territorial, en el que se aspira a crear una fuerza que actúe en toda la nación (Ornelas, 2006).

Según Hidalgo (2011; citado por Fonseca, 2014), la contrahegemonía contiene ciertos elementos:

(...) la conciencia política autónoma en las clases populares; un interés general y bloque social alternativo; superación de la perspectiva economicista y uniclasista en el proyecto político; la construcción de contra hegemonía implica la articulación entre movimientos sociales, intelectualidad crítica y proceso político (p. 267).

Se desliga del concepto de hegemonía la matriz de contrahegemonía, cuyos elementos se basan en la construcción de la conciencia política y autónoma en las clases y sectores populares. Además, se considera fundamental un proceso político que transite hacia un bloque social alternativo, para que los escenarios de disputa den paso hacia el logro y consolidación de los intereses generales de la sociedad (Lester, 2001).

Como lo afirma Campione (2005), no existe una conciencia "espontanea” generada en el proceso de producción; las clases subalternas sólo pueden llegar a las fases superiores de su desarrollo si se logra una autonomía frente a las clases dominantes, a través de la adhesión de otros grupos políticos aliados. Por eso, una vez que se desarrolla la contrahegemonía, la cual cuestiona la visión del mundo, se logrará implementar el espíritu de distinción y escisión existente en toda la sociedad.

De manera consecuente, una de las formas en las que el espectro contrahegemónico se empezó a desarrollar en América del Sur, fue el proceso de unificación que experimentaron los pueblos que históricamente enfrentaron el dominio colonial, liberal, nacionalista y neoliberal, éstos, han planteado una reestructuración económica y estatal con el objetivo de conseguir el reconocimiento del derecho de autodeterminación y territorialidad (Tapia, $2011)$.

La aparición de la hegemonía alternativa o contrahegemonía se deriva de la posibilidad de que el grupo subalterno se pueda convertir en hegemón, pasando del plano económico-corporativo al ético-político; de esa manera, los intereses deben ser presentados sobre un plano universal, que debe tener como base la esfera económica y corporativa. En contraposición a lo afirmado por Gramsci 
(1971; citado por Salvadori, 1981), Campione (2005) afirma la necesidad de partir de un plano que no incluya intereses económicos o se debe tener en cuenta los fundamentos del predominio del ámbito económico sobre la minoría explotadora, únicamente como un factor a morigerar por quienes aspiran a configurar un bloque histórico.

En efecto, la contraofensiva suramericana a los intereses foráneos de la región en las últimas décadas, se caracteriza por articular el marco económico, político y social dentro del institucionalismo regional, de modo que los países del subcontinente pudieran constituir un bloque alternativo exento de la trayectoria dependentista que se venía desarrollando. En efecto, la matriz del discurso en Sur América definió a su enemigo histórico: Estados Unidos.

Según Tapia (2011), en la región se pueden determinar dos periodos específicos en los que se conformó el trasfondo contrahegemónico: el primero, se refiere a la época de la creación y el impulso de los bloques históricos de los movimientos obreros y los proyectos socialistas; el segundo, se configura a partir de los procesos de articulación y movilización de la política a partir de los grupos indígenas. Cada uno con la intención de hacer contraposición a las estructuras políticas y económicas del sistema burgués y neocolonial.

El argumento principal de los líderes suramericanos se basa en el proyecto imperialista de dominación implementado por la potencia, a partir de ello, según Narvaja (2012) se ha iniciado "una contraofensiva histórica y retrograda con el propósito de revertir la unión, la soberanía y la democracia de nuestro continente" (p.45). Para establecer la contrahegemonía en términos de integración suramericana Ornelas (2006) plantea que "las alianzas están dictadas por la construcción unitaria, en una lógica de unidad para la acción y teniendo como horizonte la creación del sujeto capaz de hacer frente a las fuerzas hegemónicas" (p. 107).

La Unasur puede ser planteada como una estrategia política para hacerle frente a la influencia de Estados Unidos en la región. Para confirmar esta hipótesis es necesario plantear la noción conceptual y teórica de hegemonía y contrahegemonía. Según Gramsci (1971; citado por Lester, 2001) el concepto de hegemonía se basa en "las condiciones del Estado moderno donde una clase mantiene su dominio mediante la organización especial de la fuerza y sus intereses estrechos y corporativos, de ejercer un liderazgo moral e intelectual y de realizar compromisos con una variedad de aliados que se unifiquen en un bloque popular" (p. 22). A partir de lo anterior, la lucha por la hegemonía se realiza ante la 
consolidación de un Estado rodeado por una sociedad civil fortalecida, en la cual carece de un proceso de largo alcance en términos ideológicos, lo cual implica una reforma en las conciencias y métodos de conocimientos.

Como lo afirma Pereira (2010) las futuras decisiones mundiales y cambios que seguramente ocurrirán en el escenario global contarán con la participación de América del Sur (p. 422). Unasur significa el rescate de la identidad suramericana en un proyecto que permitirá la defensa de los intereses comunes, trabajando para fortalecer un orden internacional multilateral y democrático. Este momento es de cambio en el escenario internacional y de América del Sur, debe aprovecharse para conformar una nueva etapa en las relaciones internacionales.

\section{Conclusiones}

En América Latina, bajo la noción de la Teoría de la Dependencia, se le había otorgado a los procesos de integración un enfoque netamente económico (regionalismo pos- hegemónico), el cual asociaba el regionalismo a los procesos de globalización. En la actualidad, la integración regional en América Latina posee una relación entre los ejes: regionalismo abierto, revisionista y antisistémico.

La decadencia del regionalismo abierto a finales de los 90's -que se basa en una agenda de liberalización comercial y una óptica economicista- se debe al posicionamiento de la izquierda política en muchos gobiernos de la región, los cuales rechazaron los esquemas económicos tradicionales, la debilidad institucional y el estancamiento del comercio.

Uno de los factores decisivos para la aparición de nuevos proyectos políticos ha sido la creación de la nueva agenda de seguridad de Estados Unidos, derivada de los acontecimientos del 11 de septiembre del 2001, en la que América Latina queda en un segundo plano. Dicha desatención genera autonomía en los gobiernos en materia económica, de seguridad y de desarrollo en cada país.

Los inicios de la Unasur se remontan al año 2000, a partir de la necesidad de establecer una continuidad geográfica y una comunidad de valores que concurrieran en una agenda común de desafíos y oportunidades, lo cual se materializó con la Declaración de Cusco de 2004, en el marco de la III Reunión de Presidentes de América del Sur.

La Unasur se basa en iniciativas económicas y políticas, las cuales incluyen la integración geopolítica por medio del fortalecimiento regional de las 
comunicaciones, rutas, energía y acuerdos de libre comercio que, a su vez, vinculan otros procesos de integración como Mercosur y la CAN con el objetivo de cooperar en la búsqueda de la superación de la pobreza y la desigualdad.

El proyecto integracionista de Unasur forma parte de las nuevas dinámicas de la región latinoamericana. Es decir: del regionalismo post-liberal,-caracterizado por la inclusión de la dimensión política, social y la seguridad regional- la implementación de políticas distanciadas de la liberalización comercial, el rol mayoritario del Estado en los mercados frente al sector privado, la búsqueda de independencia en términos políticos, de desarrollo y económicos frente a la política exterior hegemónica, el fomento de una agenda de cooperación en áreas distintas a la comercial y la legitimación social de los procesos integradores.

Unasur, significa el rescate de la identidad suramericana en un proyecto que permite la defensa de los intereses comunes, trabajando para fortalecer un orden internacional multilateral y democrático. Este organismo es 'la primera alternativa netamente latinoamericana que intenta ir más allá de lo económico y da un paso más allá hacia lo político y geoestratégico’.

Sin embargo, Unasur se enfrenta al desafío de constituir un bloque geopolítico relevante y capaz de hacer efectivo el proyecto de multilateralidad. Lo anterior, por medio de la unión de intereses y la convergencia de acciones concretas por parte de los Estados miembros. Esto, porque desde su creación, Unasur ha tenido dificultades concernientes a la división ideológica en la región y a los fracasos pasados de otros proyectos de integración emprendidos en América del Sur.

La Unión Suramericana de Naciones ha sido definida por Cano Linares (2010; citado por Insignares, 2013) como la "integración de la integración", su objetivo ha sido vincular procesos de integración existentes por medio de criterios de convergencia basados en proyecciones sociales y valores culturales. Es decir, Unasur es el resultado de la suma entre "el acuerdo comercial entre la Comunidad Andina y Mercosur, además de la integración física en la región suramericana y la proyección de la integración energética" (Cano, 2010, p.1).

\section{Referencias}

Alcoceba, A., (2010). La lucha contra la delincuencia organizada en los procesos de integración latinoamericanos, Guía del espacio iberoamericano de paz, seguridad y defensa, 117-126. 
Behar, J. (2001). Integración regional de América Latina Procesos y Actores. Suecia: Editores e Instituto de Estudios Latinoamericanos, Universidad de Estocolmo

Bonilla. (2010). Un nuevo regionalismo sudamericano. conos. Revista de Ciencias Sociales,(38), 23-28.

Borda, S. (2012). Desafíos y oportunidades de la Unión de Naciones Suramericanas- UNASUR. Coordinadora Regional de Investigaciones Económicas y Sociales, (18). Recuperado de http://www.cries.org

Briceño, J. (2013). Ejes y modelos en la etapa actual de la integración económica regional en América Latina. Estudios Internacionales, 175(45), 9-39.

Cano, M. A. (2010). La Unión Suramericana de Naciones: un ambicioso e innovador proceso de construcción de integración regional. Revista Electrónica Iberoamericana, 10-37.

Campione, D. (2013). Hegemonía y contra hegemonía en la América Latina de hoy: apuntes sobre una nueva época. RevistaSociohistorica, 17(18), 1-24.

Chávez, C. (2010). La inserción internacional de Sudamérica: la apuesta por la Unasur. Iconos Revista de Ciencias Sociales, (38). Recuperado de: https:// www.flacso.edu.ec

Diamint, R. (2013). Regionalismo y posicionamiento suramericano: Unasur y Alba. Revista CIDOB d'afers internacionals, (101). Recuperado de: http:// www.cidob.org.

Ferrari, F. (2014). A regional arrangement proposal for the Unasur. Brazilian Journal of Political Economy, 34(136), 413-432.

Fonseca, M. (2014). La ciudadanía en los movimientos contrahegemónicos: una aproximación desde el sur. Revista Sociológica de pensamiento crítico INTERSTICIOS, 8(1). Recuperado de http://www.intersticios.es

Gomes, M. (2012). Procesos de integración de América del Sur y el papel de Brasil: los casos del Mercosur y la Unasur. Revista CIDOB d'afers internacionals, 97(98), Recuperado de http://www.cidob.org 
Gratius S. (2013). El triángulo atlántico: arquitecturas multilaterales y reajuste de poder entre nuevas y viejas potencias". Pensamiento Iberoamericano, (8), $5-21$.

Haas, E. B. (1958). The Uniting of Europe: Political, Social, and Economic Forces 1950-57. Stanford, CA: Stanford University Press.

Insignares (2013). La Unasur: cintegración regional o cooperación política? Revista de Derecho, 40.

Lester, J. (2001). The contemporanyrelevante of Gramsci in the counter- hegemonic struggle. II Seminario de la International GramsciSociety- Brasil.

Martínez, O. (2006). Alba y Alca: El dilema de la integración o la anexión. Revista del Banco Central de Cuba, (3), 1-9.

Martínezgarnelo, G. (2013). Unasur: El proceso en construcción de la Integración Sudamericana; perspectivas, logros y desafíos. Recuperado de: http:// eprints.ucm.es/32822

Miranda, S. (2011). UNASUR: una respuesta transnacional a los nuevos retos de la seguridad en Suramérica. Recuperado de http://www.caei.com

Morra, V. (2007). O Regionalismo pos-liberal na América do Sul: origens, iniviativas e dilemas. Santiago de Chile: CEPAL.

Narvaja. E. (2012). Unasur y sus discursos: Integración regional amenaza externa y Malvinas 1 ed.- Buenos Aires, Argenitna: Editorial Biblos.

Navarro, V. R. (2013). La creación de Unasur como indicio de transformación de la hegemonía estadounidense en América del Sur. Recuperado de http://repository.urosario.edu.co

Ornelas, R. (2006). Contrahegemonías y emancipaciones. Apuntes para un inicio de debate. Los desafios de las emancipaciones en un contexto militarizado, 95121.

Pereira. C. (2010). La aportación de la Unasur para el surgimiento de América del Sur como actor global de relevancia en el escenario internacional (2004-2008). (Tesis Doctoral). Universidad Complutense, Madrid, España 
Rigozzi, P. y Diana T. (abril, 2011). The rise of pot- hegemonic regionalism. The case of Latin America. LATIN Working Paper (130).

Rigirozzi, P. (2010). Region, Regioness and Regionalism in Latin America. Towards a new Synthesis, LATN, (130).

Rusell, R. (2011). América Latina: ¿entre la integración y la polarización? Un falso dilemma. Agenda internacional de América Latina: entre nuevas y viejas alianzas. Buenos Aires

Salvadori, M (1981) Gramsci y el PCI. Dos concepciones acerca de la hegemonía, En: $A A V V$, Revolución y democracia en Gramsci. Barcelona: Fontamara.

Sanahuja, J. A. (2007). Regionalismo e integración en América Latina: balance y perspectivas. Pensamiento Iberoamericano, O. Recuperado de https:// dialnet.unirioja.es.

Sanahuja, J. A. (2010). Del "regionalismo abierto" al "regionalismo post- liberal”. Crisis y cambio en la integración regional en América Latina. Anuario de la integración regional de América Latina y el Gran Caribe 2008-2009, (9), 1-523.

Saraiva, M. G. (2012). Procesos de integración de América del Sur y el papel de Brasil: los casos del Mercosur y la Unasur. Revista CIDOB d'afers internacional, (97), 87-100.

Unión de Naciones Suramericanas. (s.f.). ¿Cómo funciona Unasur? Recuperado de http://www.unasursg.org.

Vivas-Eugui, D. (2003). Regional and bilateral agreements and a TRIPS-plus world: the Free Trade Area of the Americas (FTAA).Geneva: Quaker United Nations Office, 1,1-36.

Tapia, L. (2011). La configuración de un horizonte contrahegemónico en la región andina. Utopía y Praxis Latinoamericana, 16(53), 121-123. Recuperado de: http://www.produccioncientificaluz.org/index.php/utopia/article/ view/3423/3422.

Bermúdez, C. (2011). La integración regional a comienzos del siglo XXI: MERCOSUR y UNASUR. Memorias, 8(14), 222-223. Recuperado de: http:// www.scielo.org.co/pdf/memor/n 14/n 14a09.pdf 